\title{
Development of an easy-to-handle murine model for the characterization of radiation-induced gross and molecular changes in skin
}

\author{
Hsien Pin Chang ${ }^{1}$, Jae Ho Cho ${ }^{2}$, Won Jai Lee ${ }^{1}$, Hyun Roh $^{1}$, Dong Won Lee ${ }^{1}$ \\ ${ }^{1}$ Department of Plastic and Reconstructive Surgery, Institute for Human Tissue Restoration and ${ }^{2}$ Department of Radiation Oncology, Yonsei \\ University College of Medicine, Seoul, Korea
}

Background Radiation-induced skin injury is a dose-limiting complication of radiotherapy. To investigate this problem and to develop a framework for making decisions on treatment and dose prescription, a murine model of radiation-induced skin injury was developed.

Methods The dorsal skin of the mice was isolated, and irradiation was applied at single doses of 15, 30, and 50 Gy. The mice were followed for 12 weeks with serial photography and laser Doppler analysis. Sequential skin biopsy samples were obtained and subjected to a histological analysis, immunostaining against transforming growth factor beta (TGF- $\beta$ ), and Western blotting with Wnt-3 and $\beta$-catenin. Increases in the levels of TGF- $\beta$, Wnt, and $\beta$-catenin were detected after irradiation.

Results All tested radiation doses caused progressive dermal thickening and fibrosis. The cause of this process, however, may not be radiation alone, as the natural course of wound healing may elicit a similar response. The latent appearance of molecular and histological markers that induce fibrosis in the 15 Gy group without causing apparent gross skin injuries indicates that $15 \mathrm{~Gy}$ is an appropriate dose for characterizing the effects of chronic irradiation alone. Thus, this model best mimics the patterns of injury that occur in human subjects. Conclusions This animal model can be used to elucidate the gross and molecular changes that occur in radiation-induced skin injury and provides an effective platform for studying this adverse effect without complicating the process of wound healing.

Keywords Radiation / Wound and injuries / Skin / Mice
Correspondence: Dong Won Lee Department of Plastic and Reconstructive Surgery, Yonsei University College of Medicine, 50 Yonsei-ro, Seodaemun-gu, Seoul 03722 , Korea

Tel: +82-2-2228-2215

Fax: +82-2-393-6947

E-mail:xyphoss@yuhs.ac

Received: 26 Jan 2018 • Revised: 29 May 2018 • Accepted: 3 Jul 2018

pISSN: 2234-6163 • elSSN: 2234-6171 • https://doi.org/10.5999/aps.2018.00101 • Arch Plast Surg 2018;45:403-410

\section{INTRODUCTION}

Radiotherapy is an effective treatment modality that induces tumor apoptosis; however, it also causes radiation injury to the surrounding normal tissues. Skin damage is one of the most common adverse effects and is often the limiting factor in radiotherapy, and radiation injury leads to acute and chronic toxicity.
Despite refinements in the delivery of radiation therapy, cutaneous injury remains one of the major complications and limits the available therapeutic options. Exposure of the skin to ionizing radiation induces a cascade of cellular events that lead to acute and chronic effects [1]. In the acute phase, erythema may occur within hours of irradiation. Subsequently, desquamation and ulceration appear at higher doses of radiation. These acute 
events typically resolve spontaneously. However, irreversible radiation-induced wounds can occur in chronic cases [2]. Although the dangers of chronic radiation are well documented, the exact cellular mechanisms associated with radiation-induced fibrosis remain poorly elucidated. Therefore, to understand these changes and to develop a framework for making decisions on treatment and dose prescription, a reliable and reproducible animal model of cutaneous radiation injury needs to be developed.

Previously, a porcine skin injury model used to characterize the dose-dependent response to high-dose radiation was reported [3]. The study involved dividing the dorsal skin of a mini pig into four para-sections. Using a $6 \mathrm{MeV}$ electron beam, a single fraction of $15,30,50$, or 75 Gy was delivered to each section. Porcine skin has characteristics similar to those of human skin. The epidermis is thick, with a sparse hair coat, and shows similar epidermal turnover kinetics, lipid composition, and collagen and elastic fibers to humans. The healing mechanism of wounds also acts primarily by epithelization [4]. Despite the similarities in skin characteristics between humans and pigs, there are several limitations to the pig model. Pigs are substantially more expensive and their large size makes them difficult to manage. In addition, a skilled veterinarian is required to anesthetize a pig. Some reagents, such as antibodies and polymerase chain reaction arrays, are less readily available, and transgenic pigs are more difficult to produce than other transgenic animals [5].

In this study, we aimed to develop an animal model using mice. A reproducible and easy-to-handle model with wide availability and lower cost is needed to characterize the natural development of skin damage caused by radiation. Although the porcine model has been shown to be effective in characterizing dose- and time-dependent relationships, this model is difficult to apply in a large-scale study. Given these considerations, there is a need for an easier-to-handle and more accessible model for the characterization of changes that occur in fibroblasts and epithelial cells. In 2011, a mouse model was introduced that used single-fraction radiation doses over $30 \mathrm{~Gy}$ on dorsal skin to observe the resultant gross patterns, fibrosis, Smad3 expression, bursting strength, and elasticity [6]. This model, however, did not investigate lower radiation doses that caused chronic fibrotic changes without causing significant acute gross injuries. Herein, we report a model that mimics the gross and histological changes in the skin that occur after irradiation, mediated by common cellular pathways. Various single doses of irradiation (15, 30, and $50 \mathrm{~Gy}$ ) were delivered, and dermal thickening, fibrosis, and the levels of expression of transforming growth factor beta (TGF- $\beta$ ), Wnt-3a, and $\beta$-catenin were observed during the course of 12 weeks.

\section{METHODS}

\section{Mouse skin irradiation}

All animals were housed in a facility accredited by the Association for Assessment and Accreditation of Laboratory Animal Care, and all experimental procedures were approved by the Institutional Animal Care and Use Committee (number 20120188). In this experiment, 6- to 8-week-old BALB/C mice (OrientBio) were anesthetized using a mixture of Zoletil (30 $\mathrm{mg} / \mathrm{kg})$ and Rompun $(10 \mathrm{mg} / \mathrm{kg})$. Hair was removed from the dorsal skin using an electric clipper. After hair removal, the center area of dorsal skin, sized $1.5 \times 2 \mathrm{~cm}$, was exposed $(\mathrm{n}=10$ for each group) to a single fraction of 15,30 , or 50 Gy using an XRAD320 irradiator (Precision X-Ray, North Branford, CT, USA) with a 1-cm-thick bolus.

\section{Skin toxicity assessment}

Serial photographs of the dorsal skin surface were taken to evaluate the macroscopic appearance of the injury at 2, 4, 6, 8, and 12 weeks. Skin biopsy specimens were sampled from the zone of irradiation at 4,8 , and 12 weeks after the treatment. The samples were then fixed with $4 \%$ paraformaldehyde for 15 minutes, embedded in paraffin, and sectioned for hematoxylin and eosin (H\&E) and Masson trichrome staining. The staining solution was prepared using a standard protocol.

\section{Doppler examination}

Serial scanning of blood flow within the irradiated area was conducted using a laser Doppler perfusion imager (Periflux System 5000; Perimed AB, Jarfalla, Sweden) every 2 weeks after irradiation. The Doppler probe was used to scan the center of the exposed dorsal skin $($ dimensions $=1.5 \times 2 \mathrm{~cm})$.

\section{Immunohistochemical analysis}

Immunohistochemistry was performed against TGF- $\beta$ and type I collagen (Col I). Slides were incubated overnight at $4^{\circ} \mathrm{C}$ with anti-TGF- $\beta$ (Abcam, Cambridge, UK) and rabbit anti-Col I antibody (Abcam) diluted in 1\% blocking serum. After several stages of washing with phosphate-buffered saline (PBS), the tissue sections were incubated with peroxidase-conjugated goat anti-rabbit polyclonal antibody (DAKO, Carpinteria, CA, USA) at a dilution of 1:200 in PBS for 30 minutes at $37^{\circ} \mathrm{C}$. After several washes in PBS, the signals in the tissue samples were revealed by incubating the tissues with DAB (DAKO). Semiquantitative analyses of TGF- $\beta$ and Col I expression were performed using MetaMorph image analysis software (University Image Corp., Buckinghamshire, UK). 


\section{Western blotting}

Tissues were lysed in $50 \mathrm{mM}$ Tris- $\mathrm{HCl}(\mathrm{pH} 7.6)$ containing $1 \%$ Nonidet P-40, $150 \mathrm{mM}$ sodium chloride, and $0.1 \mathrm{mM}$ zinc acetate in the presence of protease inhibitors. Tissue lysates were separated by performing $10 \%$ sodium dodecyl sulfate-polyacrylamide gel electrophoresis and were transferred onto a polyvinylidene difluoride membrane (EMD Millipore, Billerica, MA, USA). The membrane was blocked with blocking buffer for 1 hour and incubated with primary antibodies against Wnt-3a (Santa Cruz Biotechnology, Dallas, TX, USA), $\beta$-catenin (Cell Signaling Technology, Danvers, MA, USA), and $\beta$-actin (SigmaAldrich, St Louis, MO, USA). The membranes were then incubated with horseradish peroxidase-conjugated mouse secondary antibody (Santa Cruz Biotechnology) for 2 hours at room temperature. Signals of the target proteins were detected using an electrochemiluminescence detection kit (Thermo Fisher
Scientific, Waltham, MA, USA), according to the manufacturer's instructions. Protein expression was analyzed using ImageJ software (National Institutes of Health, Bethesda, MD, USA).

\section{Statistical analysis}

Each experiment was performed in triplicate. Data are presented as mean \pm standard error of the mean. Results from the various groups were compared using one way analysis of variance, followed by the post hoc Student $t$-test for unpaired observations with the Bonferroni correction for multiple comparisons. P-values $<0.05$ were considered to indicate statistical significance.

\section{RESULTS}

\section{Gross changes of radiation-treated skin}

To determine the appropriate range of radiation dosage for char-

\section{Fig. 1. Gross photographs, blood flow and H\&E staining}

(A) Representative photographs of the skin at weeks 2,4,6,8,10, and 12 after a single radiation treatment with 15, 30, and 50 Gy doses (scale bar $=5 \mathrm{~mm}$ ). (B) Serial analysis of skin blood flow at weeks 2 to 12 measured using laser Doppler after irradiation. (C) Hematoxylin and eosin (H\&E) staining at 4,8 , and 12 weeks after irradiation (scale bar $=500 \mu \mathrm{m}$ ).
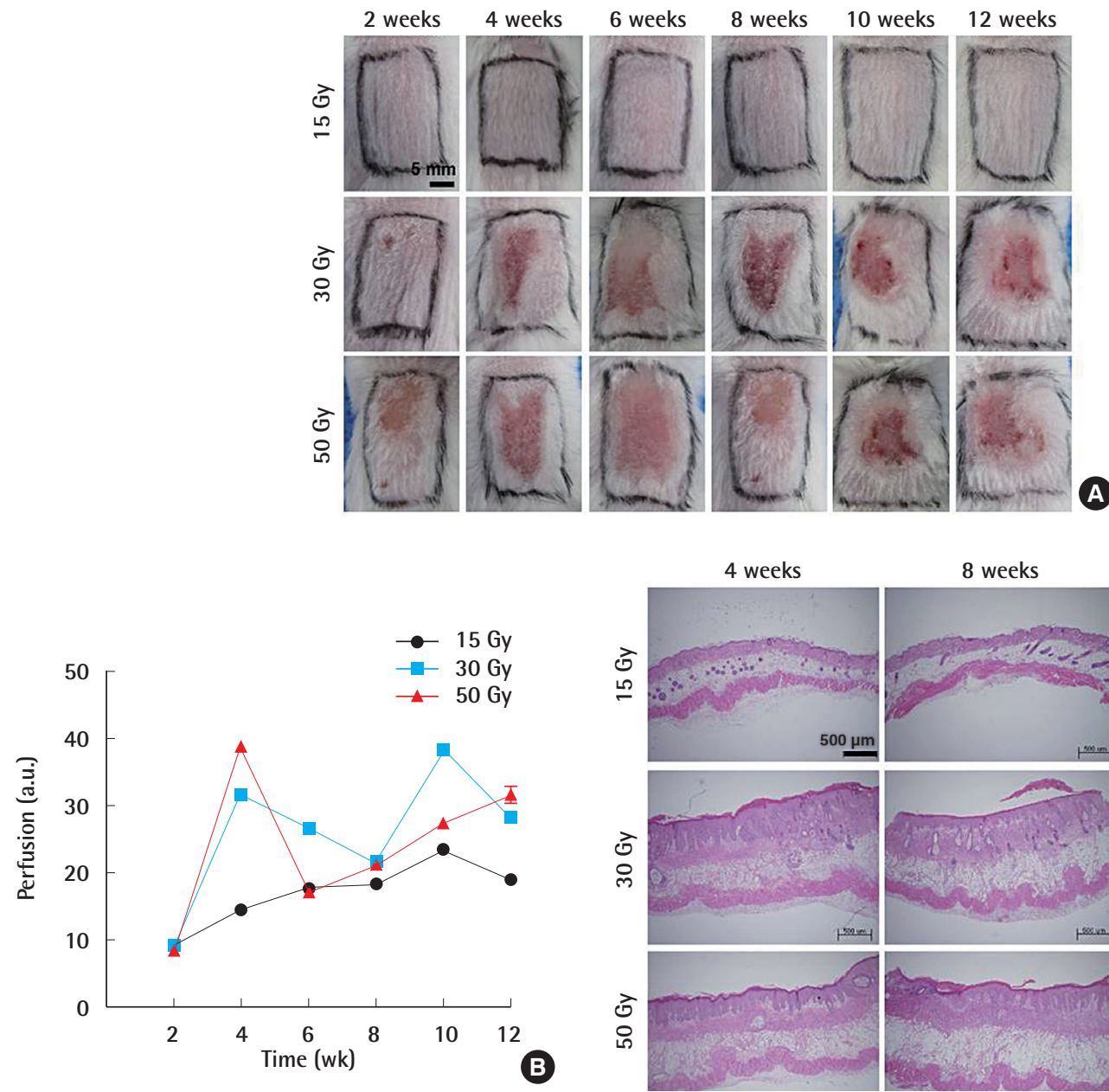

8 weeks

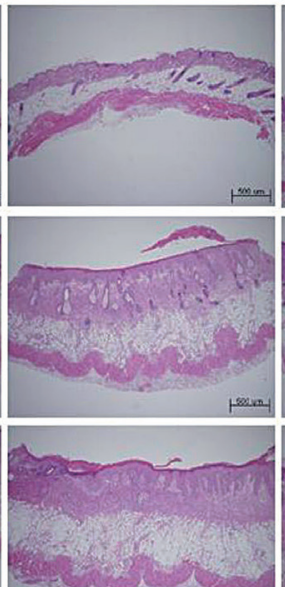

12 weeks

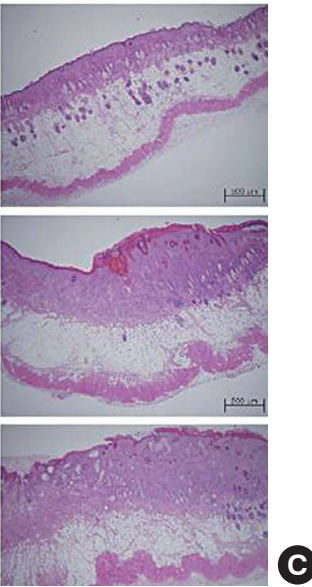


acterizing the chronic effects of radiation in a murine model, we initially conducted experiments with 30, 40, and $50 \mathrm{~Gy}$, and observed the gross skin changes for 10 weeks (Supplemental Fig. S1A). In the group irradiated with $30 \mathrm{~Gy}$, ulceration and alopecia developed at week 4 and spontaneous healing was observed at week 8 . In the 40 and 50 Gy group, ulceration occurred at week 2 and spontaneous healing occurred between weeks 8 and 10. Supplemental Fig. S1B depicts the blood flow measured using laser Doppler. In all tested groups, blood flow increased until week 4 and decreased thereafter. From week 8 onward, there were no significant differences in blood flow among all radiation dose groups. Similar observations in humans were reported in previous studies that demonstrated that irradiation induced increases in blood flow $[7,8]$. Overall, these results suggested that the 40 and 50 Gy doses might be too high, as they caused unwanted acute skin injuries, such as ulceration, as early as week 2 . To simulate the chronic effects of radiation that does not cause skin injuries in a short time span, 15, 30, and 50 Gy doses were selected for further study.

The skin changes that occurred in a time of 12 weeks are shown (Fig. 1A). In the 15 Gy group, skin ulceration did not occur throughout the 12 -week period. In the 30 Gy group, all tested mice developed ulcerations by week 4 post-injury, and spontaneous healing occurred at weeks 8 to 10 . In the 50 Gy group, all mice developed ulcerations by week 4 and spontaneous healing was observed at weeks 10 to 12 . An increasing trend in blood flow was observed in the 15 Gy group during the 12-week experimental period (Fig. 1B).

\section{Fig. 2. Fibrosis analysis and collagen quantification}

Fibrosis and collagen analysis. (A) Masson trichrome staining at weeks 4, 8, and 12 in the 15, 30, and 50 Gy dose-treated groups (scale bar $=50$ $\mu \mathrm{m})$. (B) Immunohistochemical staining against collagen type I at weeks 4, 8, and 12 in the radiation-exposed groups (scale bar $=50 \mu \mathrm{m}$ ). (C) Image-based quantification through MetaMorph image analysis.
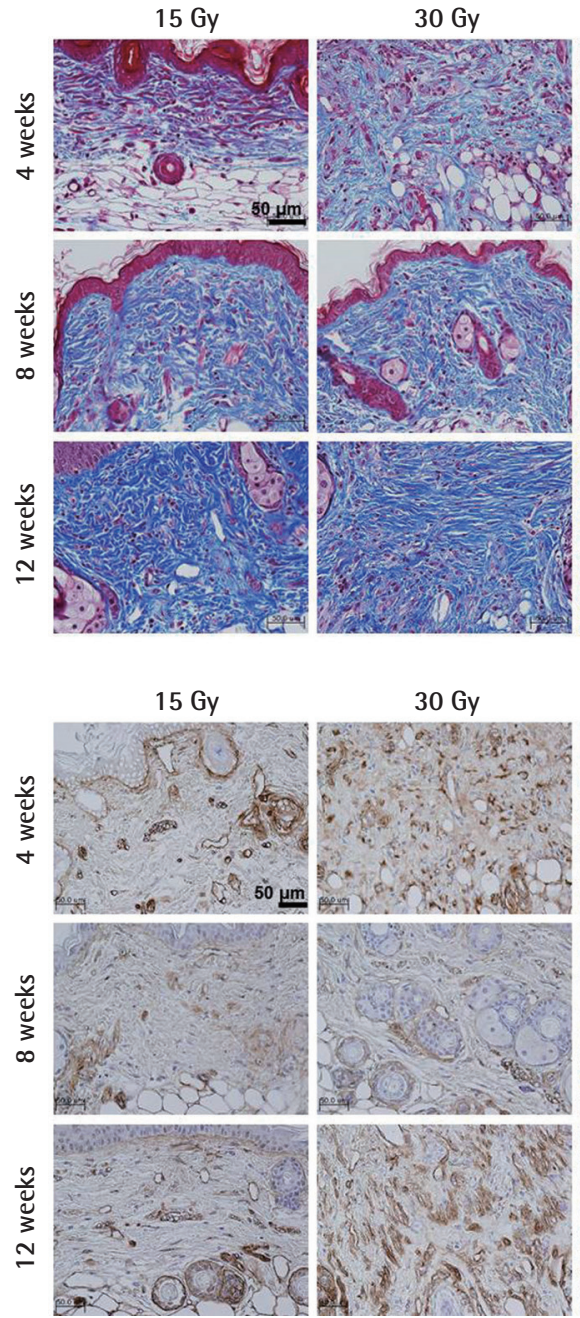

30 Gy

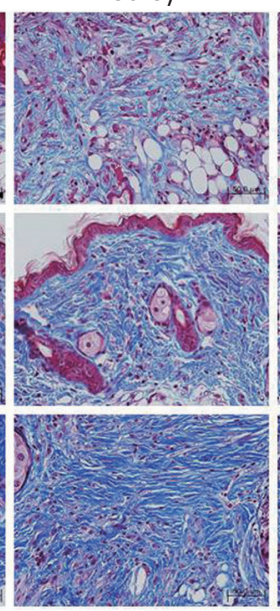

30 Gy

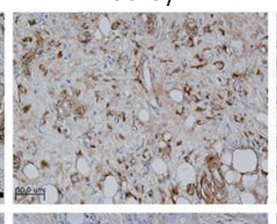

50 Gy

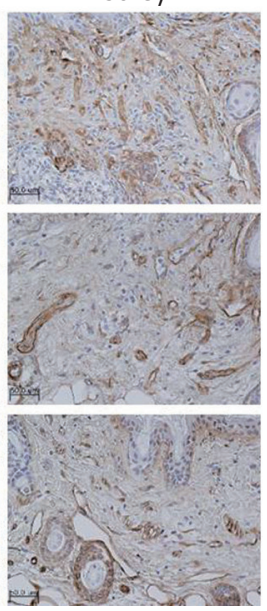

A

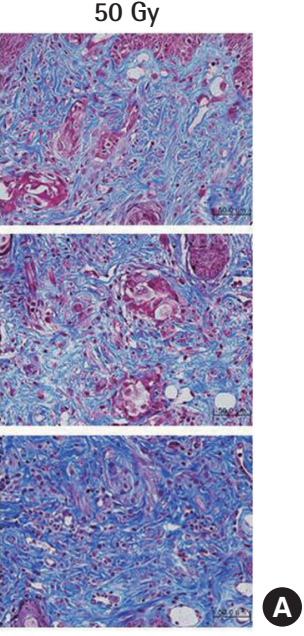

B

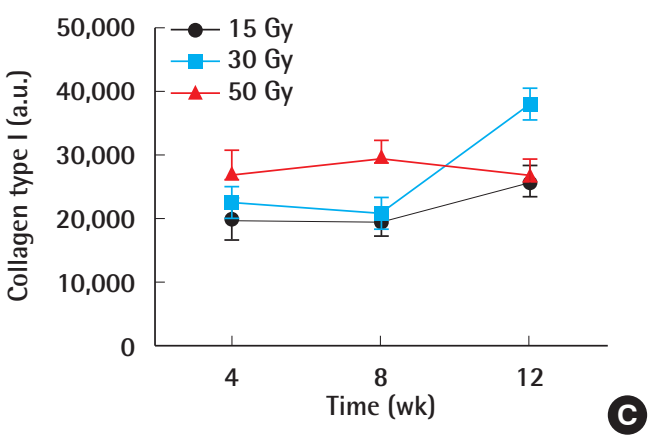




\section{Progressive dermal thickening and fibrosis after irradiation}

The histological changes that occurred at weeks 4, 8, and 12 after irradiation with the three selected radiation doses are shown (Fig. 1C). H\&E staining revealed many miniaturized follicles and a small amount of dermal thickening in the 15 Gy group, as the skin thickness remained similar throughout the 12-week period. In contrast, mice exposed to higher radiation doses (30 or $50 \mathrm{~Gy}$ ) developed progressive dermal thickening with skin desquamation that grossly presented as ulceration. As visualized using Masson trichrome staining, layers of dermal collagen fibers generally increased and thickened as the dose of radiation increased (Fig. 2A). Next, immunohistochemical analysis against Col I was performed (Fig. 2B). Immunohistochemical staining against $\mathrm{Col} I$ at week 4 in all tested groups showed greater collagen expression than in the normal group (Fig. 2C). Interestingly, similar patterns of fibrosis and collagen deposition occurred in the 15 Gy group despite the absence of ulceration in the gross photographs. Overall, these results indicate that radiation-induced fibrosis occurred in all groups, probably due to chronically activated myofibroblasts [2].

\section{Molecular changes after irradiation}

Radiation-induced skin injury is mediated through the excessive production of various cytokines, and TGF- $\beta$ plays a central role in mediating radiation-induced fibrosis [2,9-11]. The expression levels of TGF- $\beta$ were quantified using MetaMorph image analysis and the representative immunohistochemical images are shown (Fig. 3A). In the 15 and 30 Gy groups, a steady increase in TGF- $\beta$ occurred after week 8 , whereas the 50 Gy group showed a reverse trend and eventually plateaued (Fig. 3B).

To characterize the differential protein expression in the Wnt signal cascade in response to different radiation doses, the protein expression of Wnt-3a and $\beta$-catenin was analyzed using Western blot analysis (Fig. 4A). $\beta$-Catenin is a key component in the canonical Wnt-signaling pathway and has been reported to be activated during fibrosis and to mediate cellular responses to radiation [12]. In the 15 Gy group, Wnt-3a expression increased until week 8 and decreased thereafter. Generally, there was a positive correlation between the time progression and the expression level of Wnt-3. The expression of $\beta$-catenin in the 15 Gy group also showed a similar trend. The expression increased in the earlier period and decreased thereafter. From these results, it can be concluded that the Wnt and $\beta$-catenin signaling cascade was activated in the early stages, with the signal waning by week 12 , albeit through direct downstream regulation. The differential expression levels in the 15 Gy group are presented as a bar graph (Fig. 4).

\section{DISCUSSION}

The purpose of this study was to establish a reliable animal model that can be used to characterize the natural course of the gross, molecular, and histological changes that occur in skin fibrosis caused by radiation. As improvements are made in treatment modalities in the field of radiation oncology, damage to

\section{Fig. 3. TGF- $\beta$ expression}

(A) Representative immunohistochemical images of transforming growth factor (TGF)- $\beta$ (scale bar $=200 \mu m$ ). (B) Image-based quantification of TGF- $\beta$ at weeks 4,8 , and 12 in the 15, 30, and 50 Gy dose-treated groups.
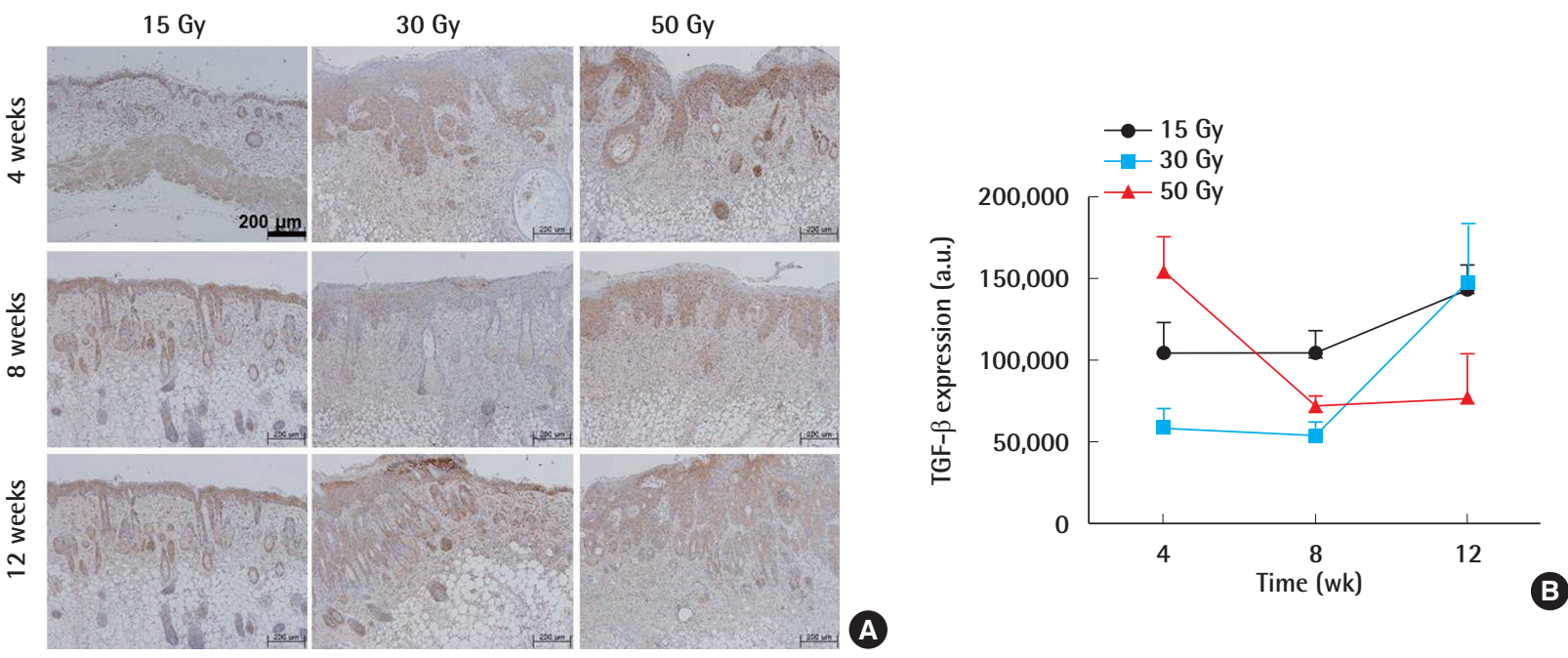


\section{Fig. 4. $\beta$-Catenin and Wht-3a expression}

(A) Representative Western blot images of Wnt-3a, $\beta$-catenin, and the housekeeping protein $\beta$-actin in skin tissue extracts in the 15 Gy group at weeks 4,8 , and 12 , and quantitative analysis of (B) Wnt-3a and (C) $\beta$-catenin expression relative to that of $\beta$-actin in each group. ${ }^{*} P<0.01$ vs. the 4-week group, $n=3$.
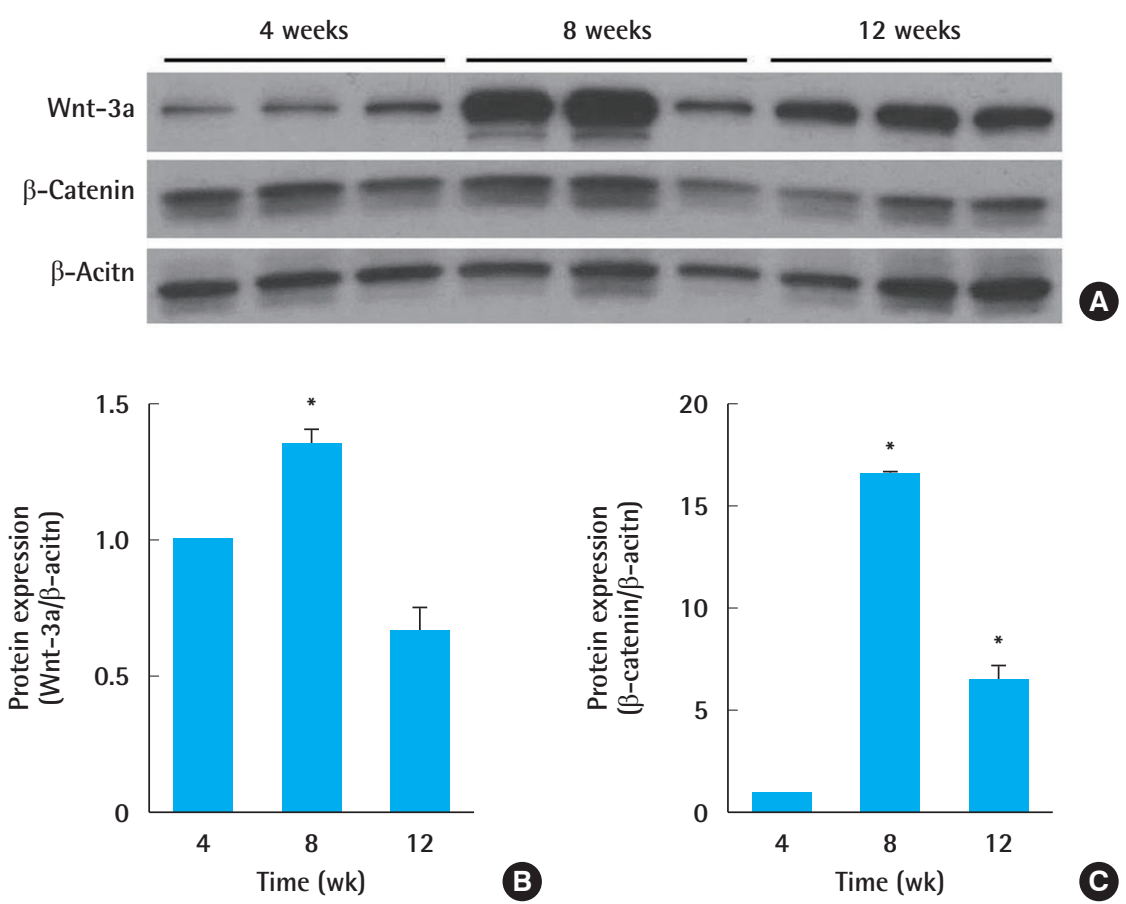

the surrounding tissues may decrease, but the overlying dermal structures remain exposed to radiation. In order to successfully develop an injury model that mimics the gross, histological, and molecular changes that occur in human skin in therapeutic contexts, it was imperative to identify a radiation dose that did not cause gross acute cutaneous injury. The latent appearance of molecular and histological markers observed in this study after a single dose of 15 Gy suggest that 15 Gy is an appropriate dose for testing the effects of chronic radiation.

Histological analysis showed that there was a dose-dependent relationship to radiation. Thickening of the skin and desquamation occurred at earlier stages with higher doses compared to the lower dose of $15 \mathrm{~Gy}$, indicating a latency period in low-dose radiation. Although a dose-dependent relationship in molecular and histological changes was established, it was more difficult to observe a meaningful outcome in the protein expression level in the Wnt signaling pathway. Nonetheless, through this model, we established that radiation caused increases in the protein expression levels of Wnt-3a and $\beta$-catenin, peaking at week 8 . This is in accordance with previous reports showing that radiation stimulated $\mathrm{Wnt} / \beta$-catenin signaling [13]. It has previously been reported that $\beta$-catenin promoted the survival of irradiated fibroblasts $[12]$. As there was a decrease in the level of both proteins in week 12 despite the accumulation of Col I seen in the histological analysis, we can conclude that the accumulation of Col I may be mediated by a different pathway after a certain time.

Unlike normal wound healing, in which feedback mechanisms tightly regulate the activation of fibroblasts into myofibroblasts, and in turn the proliferation and deposition of the collagen matrix, fibrosis is characterized by sustained activation of myofibroblasts through the abnormal production of stimulating factors [2]. One of the key stimulating factors is TGF- $\beta$. There was an overall increase in TGF- $\beta$ expression levels throughout week 12 , which reaffirms that TGF- $\beta$ plays a role in mediating radiation-induced tissue fibrosis through binding to type I/II receptor complexes, leading to activation of Smad proteins even at low doses of radiation [9]. Hence, our model incorporates two main pathways of radiation injury: one that activates $\beta$-catenin through the Wnt ligand and another mediated by the TGF- $\beta$ ligand. Attempting to mitigate radiation-induced fibrosis by preventing the sustained activation of myofibroblasts through key downstream mediators of TGF- $\beta$ and Wnt in this murine model could be an interesting future endeavor.

In this experiment, we also attempted to measure skin blood flow by using laser Doppler imaging after irradiation. We showed that there was a general increase in blood flow in re- 
sponse to all tested radiation doses. The increase in blood flow may have been due to the short experimental time frame, as previous studies reported microvascular injury due to chronic ischemia, which hampers tissue perfusion [2]. The increased perfusion seen from week 6 onwards for the 50 Gy group and week 8 onwards for the $30 \mathrm{~Gy}$ group after a rapid decrease from week 4 to 6 and week 4 to 8 for the 50 and 30 Gy group, respectively, may have been due to increased recruitment of angiogenic factors after vascular damage. Vascular damage is known to be repaired through angiogenesis and vasculogenesis. There is also increasing evidence showing that radiation causes preferential recruitment of bone marrow-derived cells, which may lead to the expression of angiogenic factors; however, the exact time frame of this process remains unclear [14]. In addition, vessel damage does not occur immediately after radiation injury. Arterioles start to be disrupted after 2 to 3 weeks of radiation, and the sequence of events leading to greater permeability of the endothelial cells and the slowing of blood flow does not occur until a few weeks after vessel damage [15]. This may possibly result in a hyperemic state, leading to increased blood flow in the very early period, as indicated by the increased perfusion on Doppler analysis at week 4 in the 30 and 50 Gy groups. This was in sharp contrast to the lower 15 Gy group. Blood flow steadily increased until week 10, which may have been due to less severe fibrotic change. Nonetheless, in order to test the effects of radiation on perfusion more accurately, further tests investigating angiogenic factors, such as vascular endothelial growth factor, may be necessary to prove the above hypothesis.

Murine models are advantageous because of their ease of handling, lower cost, and the ability to use larger numbers of animals in studies. Moreover, ample molecular reagents and probes are available that can further delineate the molecular pathways involved in progressive cutaneous changes [6]. Radiation-driven epithelial-to-mesenchymal transition using a murine model may be studied using this model as well.

There were a few limitations to this study. Despite our efforts to limit the radiation exposure to a consistent area of dorsal skin with the same dimension and position, some areas may have been exposed to a higher dose due to the curvature of the dorsal skin. In addition, there are some differences in skin structure between humans and mice. These differences may elicit a dissimilar wound healing process. For example, mice have the subcutaneous panniculus carnosus muscle, which catalyzes wound contraction and collagen formation. This muscle is non-existent in humans [16]. Despite some differences between human and murine skin, the standardization, reproducibility, ability to validate results across studies, and feasibility of studying transgenic and knockout mice make mouse models valuable for investigat- ing the effect of radiation on skin.

Herein, we established an animal model that may serve as a basis for future radiation research. We determined that radiation at a dose of 15 Gy induced an increase in biochemical markers, molecular markers such as TGF- $\beta$, and histological markers, without interfering with the process of wound healing. This enables studying the dysregulation of fibrosis through the effects of radiation alone, as fibroblasts are also activated by inflammation or through skin injury.

\section{NOTES}

\section{Conflict of interest}

No potential conflict of interest relevant to this article was reported.

\section{Ethical approval}

The study was approved by the Institutional Animal Care and Use Committee (number 2012-0188).

\section{ORCID}

Hsien Pin Chang https://orcid.org/0000-0002-2861-6371

\section{REFERENCES}

1. Collen EB, Mayer MN. Acute effects of radiation treatment: skin reactions. Can Vet J 2006;47:931-5.

2. Yarnold J, Brotons MC. Pathogenetic mechanisms in radiation fibrosis. Radiother Oncol 2010;97:149-61.

3. Kim JW, Lee DW, Choi WH, et al. Development of a porcine skin injury model and characterization of the dose-dependent response to high-dose radiation.J Radiat Res 2013; 54:823-31.

4. Seaton M, Hocking A, Gibran NS. Porcine models of cutaneous wound healing. ILARJ 2015;56:127-38.

5. Williams JP, Brown SL, Georges GE, et al. Animal models for medical countermeasures to radiation exposure. Radiat Res 2010;173:557-78.

6. Thanik VD, Chang CC, Zoumalan RA, et al. A novel mouse model of cutaneous radiation injury. Plast Reconstr Surg 2011;127:560-8

7. Inomata T, Ogawa Y, Nishioka A, et al. Changes in blood flow and skin reaction following radiation therapy. Nihon Igaku Hoshasen Gakkai Zasshi 1995;55:58-64.

8. Nitzan M, Weshler Z, Gimmon Z, et al. Irradiation induced blood flow changes in the breast during radiotherapy: a pilot study.J Basic Clin Physiol Pharmacol 1996;7:71-82.

9. Martin M, Lefaix J, Delanian S. TGF-beta1 and radiation fi- 
brosis: a master switch and a specific therapeutic target? Int J Radiat Oncol Biol Phys 2000;47:277-90.

10. Cheon SS, Wei Q Gurung A, et al. Beta-catenin regulates wound size and mediates the effect of TGF-beta in cutaneous healing. FASEB J 2006;20:692-701.

11. Poon R, Nik SA, Ahn J, et al. Beta-catenin and transforming growth factor beta have distinct roles regulating fibroblast cell motility and the induction of collagen lattice contraction. BMC Cell Biol 2009; 10:38.

12. Gurung A, Uddin F, Hill RP, et al. Beta-catenin is a mediator of the response of fibroblasts to irradiation. Am J Pathol 2009; 174:248-55.
13. Wei LC, Ding YX, Liu YH, et al. Low-dose radiation stimulates Wnt/beta-catenin signaling, neural stem cell proliferation and neurogenesis of the mouse hippocampus in vitro and in vivo. Curr Alzheimer Res 2012;9:278-89.

14. Kioi M, Vogel H, Schultz G, et al. Inhibition of vasculogenesis, but not angiogenesis, prevents the recurrence of glioblastoma after irradiation in mice. J Clin Invest 2010;120:694705.

15. Cox JD, Ang KK. Radiation oncology: rationale, technique, results. Philadelphia: Elsevier Health Sciences; 2009.

16. Dunn L, Prosser HC, Tan JT, et al. Murine model of wound healing. J Vis Exp 2013;(75):e50265.

\section{Supplementary Material}

Supplemental Fig. S1. Gross photographs and serial blood flow

(A) Representative photographs of the skin at weeks $2,4,6,8$, and 10 after a single radiation treatment with various doses. (B) Serial analysis of skin blood flow measured using laser Doppler after irradiation (perfusion units). ${ }^{*} \mathrm{P}<0.05$ vs. the 40 and $50 \mathrm{~Gy}$ group, $n=3$. (https://doi.org/10.5999/aps.2018.00101). 


\section{Supplemental Fig. S1. Gross photographs and serial blood flow}

(A) Representative photographs of the skin at weeks 2, 4, 6, 8, and 10 after a single radiation treatment with various doses. (B) Serial analysis of skin blood flow measured using laser Doppler after irradiation (perfusion units). ${ }^{*} P<0.05$ vs. the 40 and 50 Gy group, $n=3$.
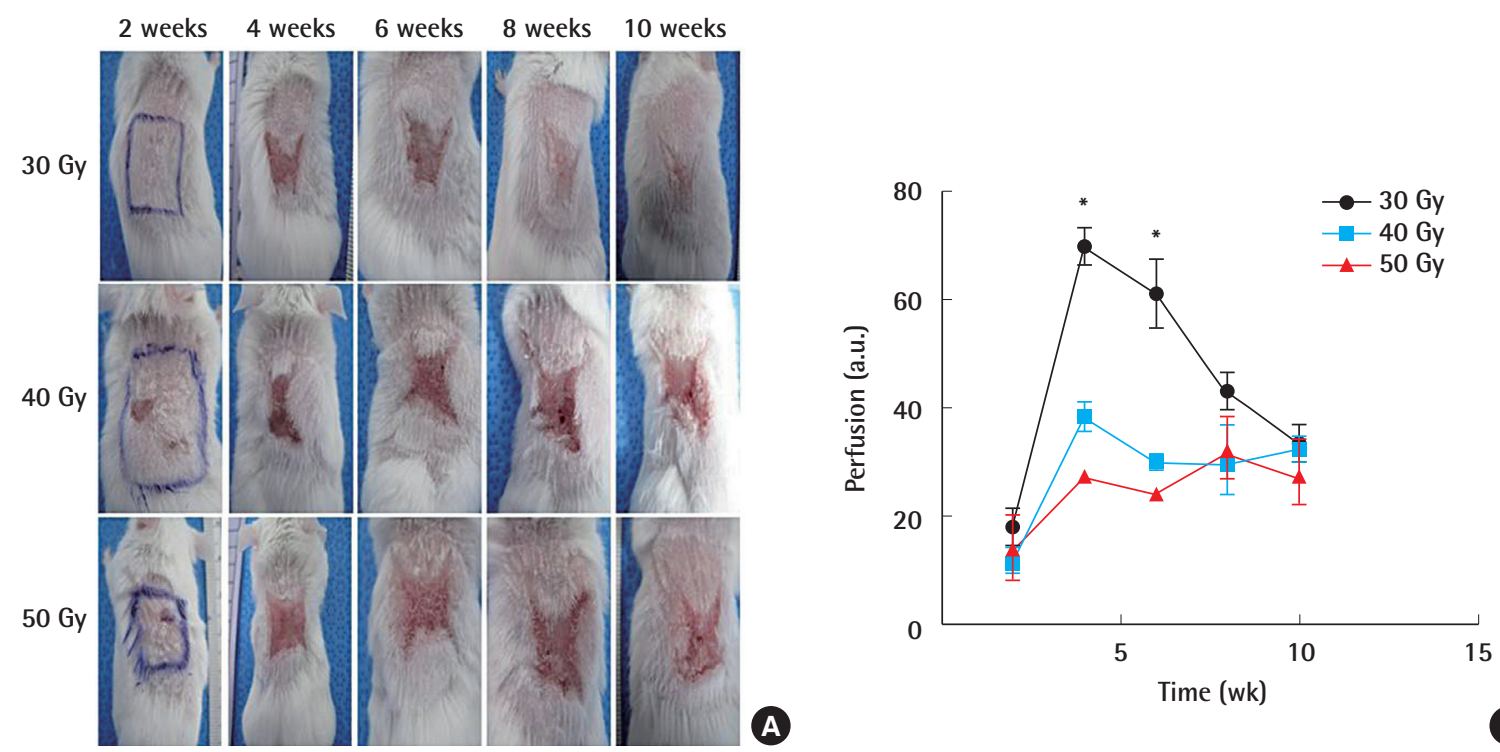